\title{
The pre-main-sequence eclipsing binary ASAS J052821+0338.5
}

\author{
H. C. Stempels ${ }^{1}$, L. Hebb ${ }^{1}$, K. G. Stassun ${ }^{2}$, J. Holtzman ${ }^{3}$, N. Dunstone ${ }^{1}$, L. Glowienka ${ }^{4,5}$, and S. Frandsen ${ }^{5}$ \\ ${ }^{1}$ School of Physics \& Astronomy, University of St Andrews, North Haugh, St Andrews KY16 9SS, Scotland \\ e-mail: Eric.Stempels@st-andrews.ac.uk \\ 2 Physics and Astronomy Department, Vanderbilt University, Nashville, TN 37235, USA \\ Astronomy Department, New Mexico State University, Las Cruces, NM 88003, USA \\ ${ }^{4}$ Nordic Optical Telescope, Apartado 474, 38700 Santa Cruz de La Palma, Spain \\ 5 Department of Physics and Astronomy, Århus University, Denmark
}

Received 31 August 2007 / Accepted 30 January 2008

\section{ABSTRACT}

\begin{abstract}
Aims. In this paper we present the first results of a detailed spectroscopic and photometric analysis of the $V=11.7^{\mathrm{m}}$ eclipsing binary ASAS J052821+0338.5.

Methods. With the FIES spectrograph at the Nordic Optical Telescope, we obtained a series of high-resolution spectra $(R \approx 47000)$ covering the entire orbit of the system. In addition we obtained simultaneous broadband photometry from three small aperture telescopes. From these spectroscopic and photometric data we have derived the system's orbital parameters and determined the fundamental stellar parameters of the two components.

Results. Our results indicate that ASAS J052821+0338.5 is a K1/K3 pre-main-sequence eclipsing binary, with component masses of $1.38 M_{\odot}$ and $1.33 M_{\odot}$ and a period of 3.87 days, located at a distance of $280 \pm 30 \mathrm{pc}$. The kinematics, physical location, and the evolutionary status of the two stars suggest that ASAS J052821+0338.5 is a member of the $\sim 11$ Myr old Orion OB1a subassociation. The systems also exhibits smooth $\sim 0.15^{\mathrm{m}}$ out-of-eclipse variations that are similar to those found in RS CVn binaries. Furthermore, the parameters we derived are consistent with the 10-13 Myr isochrones of the popular Baraffe stellar evolutionary models.
\end{abstract}

Key words. stars: pre-main sequence - stars: binaries: eclipsing - stars: binaries: spectroscopic - stars: fundamental parameters stars: individual: ASAS J052821+0338.5

\section{Introduction}

The advantageous geometry of detached double-line eclipsing binary systems makes it possible to measure stellar radii and masses to very high accuracy (1-2\%, Andersen 1991). Such accurate parameters are of crucial importance in providing observational constraints on stellar evolutionary models. However, very few of the known double-lined eclipsing binaries are stars at early stages of evolution, leaving the models in this domain with only few empirical points of reference.

The list of known double-lined eclipsing binaries with components that are in the pre-main-sequence (PMS) phase of evolution is not long. Up to now, only five low-mass doublelined eclipsing binaries in which both components are PMS objects have been reported, RXJ 0529.4+0041A (Covino et al. 2000, 2004), V1174 Ori (Stassun et al. 2004), the brown dwarf eclipsing binary 2MASS J05352184-0546085 (Stassun et al. 2006, 2007) and, very recently, JW 380 (Irwin et al. 2007b) and Par 1802 (Cargile et al. 2007). In addition, EK Cep and TY CrA (Popper 1980; Popper 1987; Andersen 1991; Casey 1998) are known to harbour one PMS component and one main-sequence component.

In this paper, we announce the discovery that ASAS J052821+0338.5 (also known as GSC2.2 N300311274, 2MASS 05282082+0338327 and USNO-B1.0 0936-0073790) is a PMS eclipsing binary system. In addition, the system could be associated with the ROSAT X-ray source

* Based on observations made with the Nordic Optical Telescope.
1RXS J052820.4+033823, located only 11" away, within ROSAT's positional error margin of $14^{\prime \prime}$. Using new highcadence photometric and high-resolution spectroscopic observations we have determined the system's fundamental properties and confirm the PMS nature of both components.

\section{Observations}

\subsection{Photometry}

ASAS J052821+0338.5 was first identified in the All-Sky Automated Survey (ASAS, Pojmanski 2002) as a detached eclipsing binary system with a maximum, out-of-eclipse $V$-band magnitude of $V=11.7^{\mathrm{m}}$ and a period of $P=1.9364$ days. In addition, the target is listed in several large photometric databases consolidated in the Naval Observatory Merged Astronomical Dataset (NOMAD-1.0, Zacharias et al. 2004), which provide optical and infra-red magnitudes of $B=12.68^{\mathrm{m}}, J=9.636^{\mathrm{m}}$, $H=9.098^{\mathrm{m}}$ and $K=8.961^{\mathrm{m}}$.

In the ASAS survey, $171 \mathrm{~V}$-band measurements of the object were obtained during the period February 2001-April 2006 with a median sampling rate of $0.25 \mathrm{~d}^{-1}$. The resulting lightcurve exhibits periodic eclipses with a depth of $\sim 0.3^{\mathrm{m}}$ and scatter in the out-of-eclipse phases with an rms amplitude of $0.15^{\mathrm{m}}$, likely due to an evolving spot configuration. We supplemented the long-term photometry from ASAS with additional precise, high-cadence observations obtained with several small aperture telescopes. 


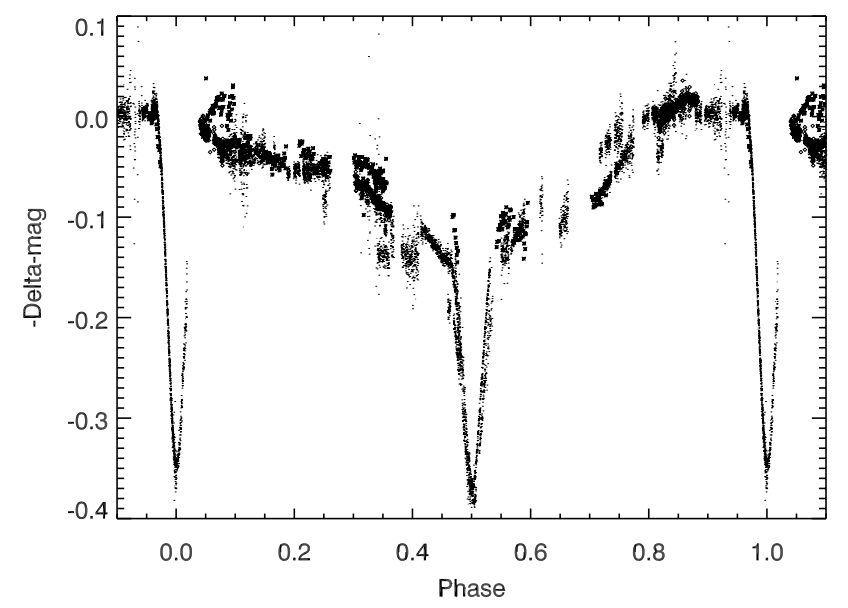

Fig. 1. All differential photometry of ASAS J052821+0338.5 collected by the NMSU-1 m, JGT-1 $\mathrm{m}$ and 3MT, phase-folded with the orbital period of 3.8729 days and epoch, $T_{0}=2454110.8500$. The error bars on the data are smaller than the plotted symbols, and estimates of the errors are given in the text.

\subsubsection{High cadence, high precision photometry}

The New Mexico State University 1-m telescope (NMSU-1m) provided the majority of the follow-up photometry. A total of $8805 \mathrm{~V}$-band measurements of the target were acquired over 31 nights between January 10-April 17, 2007. The data were processed with standard data reduction procedures including 2D bias and overscan subtraction, flat-fielding, and aperture photometry. To derive the differential photometry lightcurve, the magnitudes of three nearby, bright stars were average combined into a non-variable reference object which was subtracted from the instrumental magnitude of the target star. On February 4, 5 , and 7, three additional nights of $V$-band photometry were obtained with the 1-m James Gregory Telescope (JGT-1 m, St. Andrews, UK). These data can be directly compared with the NMSU-1 $\mathrm{m}$ data as the same basic reduction procedures were applied to the images and the same three reference stars were used to derive the differential photometry. The $V$-band differential photometry is normalized so that the magnitude of the phase between 0.94-0.96 (before the primary eclipse) has a median value of zero.

Time-series photometry in a redder waveband was obtained with the $0.5 \mathrm{~m}$ 3-Mirror Telescope (3MT; Cambridge, UK) from January-March, 2007. The filter (RG630) plus the CCD efficiency curve for the detector selects a broadband wavelength region which is between the Johnson $R$ and Cousins $I$-band filters. These data were reduced in a standard way with a version of the Cambridge Astronomical Survey Unit (CASU) data reduction and photometry pipeline (Irwin \& Lewis 2001; Irwin et al. 2007a) modified for the 3MT. These data are normalized to match the $V$-band photometry, so that the phases before the primary eclipse have a median value of zero.

The final differential photometry lightcurve of all telescopes folded with the ephemeris derived below is shown in Fig. 1. In Fig. 2 we show the original ASAS photometry (top panel) as well as our small-telescope data, divided into panels with photometry obtained within consecutive 2-4 week time intervals, the typical timescale for a stable starspot configuration. The ASAS photometry has a precision of $\sim 0.05^{\mathrm{m}}$. The small-telescope $V$-band photometry (crosses) has a sampling of $<1 \mathrm{~min}$, and has an uncertainty ranging from $\sim 0.003$ to $0.010^{\mathrm{m}}$, mainly due to variable systematic errors such as changing sky

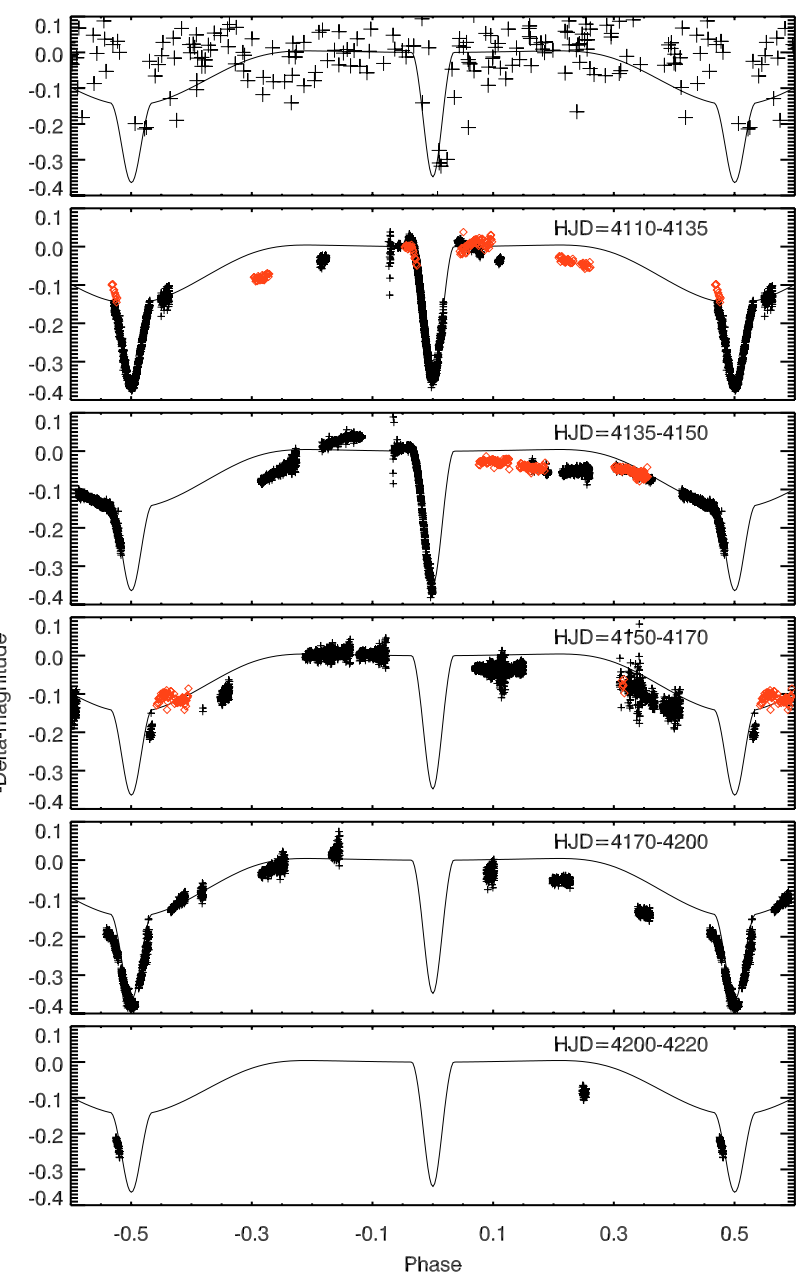

Fig. 2. This figure illustrates the evolution of out-of-eclipse variability on ASAS J052821+0338.5 during the four months (January-April, 2007) following the spectroscopic observations. The original ASAS photometry is shown in the top panel. Subsequent panels show the $V$-band lightcurve obtained with the NMSU-1 m and JGT-1 m telescopes (black crosses) as well as the red (RG630) lightcurve obtained with the $3 \mathrm{MT}$ (grey diamonds). The data are broken into 2-4 week intervals. The time span (in HJD - 2450000 ) covered by the data in each panel is given in the top right hand corner. The solid line is a simple one-spot model, plotted as a reference.

transparency. We opted to exclude 17 measurements which had unusually large photometric errors of $>0.05 \mathrm{mag}$. The red wavelength data from the 3MT (diamonds) has a precision of $0.006-0.008^{\mathrm{m}}$ and has a slightly higher scatter and lower sampling due to the smaller aperture telescope used.

The lightcurve shows deep primary and secondary eclipses $\left(\sim 0.37^{\mathrm{m}}\right)$, a period $(P \approx 3.87$ days) twice what was determined from the ASAS photometry alone, and large-amplitude out-ofeclipse variability $\left(\sim 0.15^{\mathrm{m}}\right)$. The out-of-eclipse variations are present for the duration of our four month observing campaign, but they are not completely stable. There are small variations in the lightcurve and in the depth of the secondary eclipse (approximately $\pm 0.01^{\mathrm{m}}$ ), probably caused by an evolving spot configuration (see also the discussion in Sect. 4). The observed outof-eclipse variability is qualitatively consistent with a large cool spotted region on the back side of the primary star causing the attenuation of the secondary eclipse. Also, the secondary eclipse in the ASAS data appears to be only half the depth that it exhibits in the NMSU-1m data. This is a further suggestion that the 


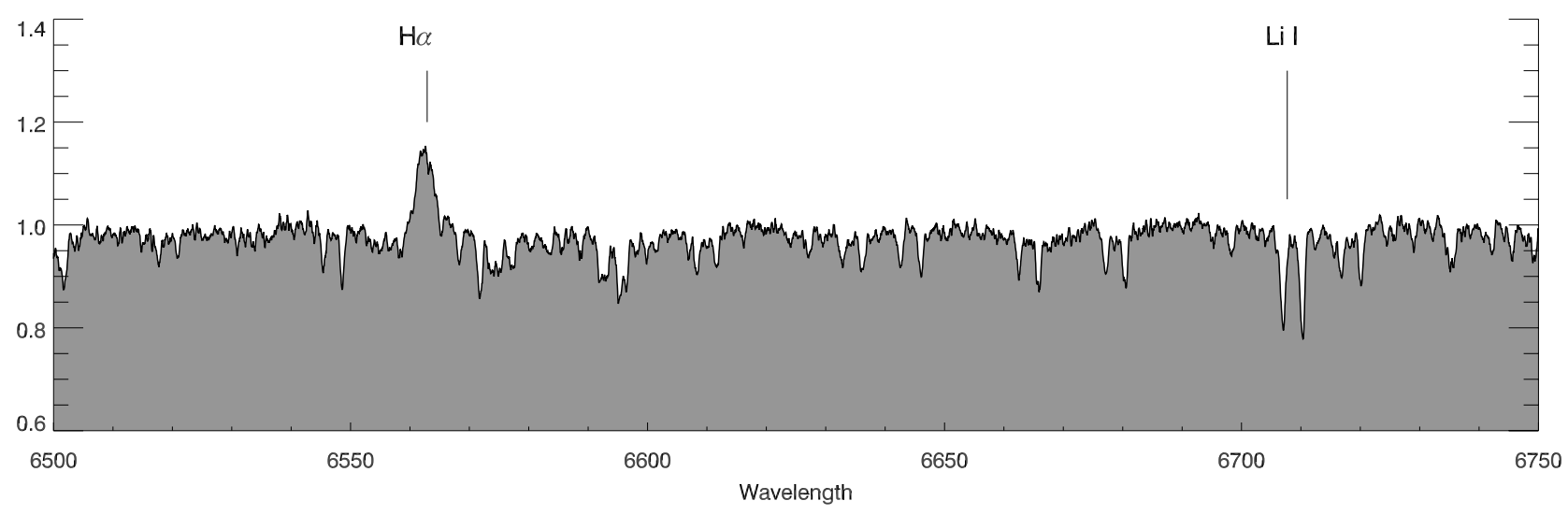

Fig. 3. This spectrum of ASAS J052821+0338.5 was obtained at phase 0.64, and shows clearly the double-lined nature of the spectrum, the emission in $\mathrm{H} \alpha$ as well as the strong absorption in the Li I 6708 line.

total depth of the secondary eclipse is being affected by variable starspots.

\subsubsection{Ephemeris}

Partial primary and secondary eclipses which were detected in the NMSU-1m data were used in combination with the ASAS photometry to derive an ephemeris for the system. We defined the epoch of the system, $T_{0}$, to be the midpoint of the most complete primary eclipse. We used the NMSU-1m data obtained on HJD $=2454110$ which contains the only highly time sampled primary eclipse showing the eclipse minimum to determine $T_{0}$. We modelled the shape of this eclipse with a 4th order polynomial and performed a $\chi^{2}$ minimization with respect to the model to derive the best fitting coefficents. We then set $T_{0}$ to be the midpoint of the model curve. Next, we fixed $T_{0}$ and solved for the period of the system using the nearly complete primary eclipse obtained on HJD = 2454110 and the only other partial primary eclipse (HJD $=2454116)$ in the NMSU-1 m data in combination with the complete ASAS lightcurve. We tested a grid of possible periods, phase-folding these data on each trial period, and found the period which minimized the $\chi^{2}$ of these data with respect to the model. The resulting ephemeris at the midpoint of the eclipse is given by

$T\left(V_{\min }\right)=$ HJD $2454110.8500 \pm 0.002+3.8729 E \pm 0.0004$

where $E$ corresponds to the cycle number. The uncertainties were estimated from the curvature of the $\chi^{2}$ surface around the minimum, using the standard equation $\sigma=\sqrt{2 / \nabla^{2} \chi^{2}}$. The lack of more than one primary eclipse and the relatively few ASAS data points with large errors compared to the NMSU-1m data are the main causes of uncertainties on the ephemeris. We have no additional constraints on the ephemeris and keep this fixed to the above values during the eclipsing binary modelling in Sect. 3 .

With observations of only one complete primary and secondary eclipse it is difficult to accurately determine the eccentricity of the system. However, we estimate this parameter by measuring the time of the midpoint of the complete secondary eclipse obtained on HJD $=2454114$ with respect to $T_{0}$ by modelling the shape of that eclipse as a 4 th order polynomial and minimizing the $\chi^{2}$. We find the timing of the secondary eclipse is marginally consistent with a circular orbit for the binary within the quoted uncertainties on the ephemeris. Therefore, we adopt an eccentricity $e=0$ for the system. However, additional highly time-sampled observations of multiple primary and secondary eclipse minima are necessary to derive a more accurate ephemeris, and determine whether there is a small but non-zero eccentricity in the system. Figure 1 shows all the small telescope photometry phase-folded with the ephemeris defined above.

\subsection{Spectroscopy}

A total of 17 spectra of ASAS J052821+0338.5 with exposure times of 15-30 min were obtained with the Fibre-fed Echelle Spectrograph (FIES) at the $2.5 \mathrm{~m}$ Nordic Optical Telescope during January $2-16,2007$. These spectra cover the wavelength range 4000-7350 $\AA$ at a resolution of $R \approx 47000$ and have a typical signal-to-noise of 30-50.

The spectra were reduced with the on-line data reduction software supplied at the telescope. This package, based on PYTHON and PYRAF was especially developed for FIES and performs all conventional steps of echelle data reduction, including the subtraction of bias frames, modelling and subtraction of scattered light, flat-fielding, order extraction, normalization (including fringe-correction) and wavelength calibration. In addition we applied the heliocentric velocity correction to the wavelength scale and performed continuum normalization.

Four additional 15-min high resolution $(R \approx 65000)$ spectra were obtained on April 5, 2007 with the UCLES spectrograph and the SEMPOL polarimeter at the Anglo Australian Telescope. These data were reduced with Echelle Spectra Reduction: an Interactive Tool (ESPRIT, Donati et al. 1997), a comprehensive reduction package for spectro-polarimetric observation.

The spectrum of ASAS J052821+0338.5 is a clear example of a double-lined spectroscopic binary. Figure 3 shows a sample of the spectrum, obtained near phase 0.64 , with well-separated components. The pre-main-sequence nature of the object is evident from the $\mathrm{H} \alpha$ emission $(E W \approx 1 \AA$, secondary only) and strong Li I 6708 absorption. Except for $\mathrm{H} \alpha$, no other emission lines are seen in the spectrum. Some of the deepest absorption lines in the spectrum, such as the $\mathrm{Mg}$ b line complex and $\mathrm{Na} 1 \mathrm{D}$ doublet, show weak emission in the line cores. There is no evidence of excess continuous emission, such as veiling.

\section{Analysis}

\subsection{Radial velocity curve}

To derive the radial velocity curve for the binary system, the 17 FIES spectra were cross-correlated against the spectrum of HD 286264, a single-lined K2IV weak-line T Tauri star, on an 
order-by-order basis using the FXCOR package in IRAF. The majority of spectra showed two distinct cross-correlation peaks, one for each component of the binary. Thus, both peaks were fit independently with a Gaussian profile to measure the velocity and velocity errors of the individual components. If the two peaks appeared blended, a double Gaussian was fit to the combined profile. For each of the 17 observations we then determined a weighted-average radial velocity for each star from all orders without significant contamination by telluric absorption features. Here we used as weights the inverse of the variance of the radial velocity measurement in each order, as reported by FXCOR. The resulting radial velocities were calibrated using the velocity offset derived by cross-correlating the template spectrum with a radial velocity standard, HD 109358 (Udry et al. 1999). In these data, we find no evidence for a third component, since the cross-correlation function showed two distinct peaks.

To each of the four UCLES spectra, we applied the technique of least-squares deconvolution (LSD, see Donati et al. 1997). This technique, often used in Doppler Imaging, allows us to construct, with a very high signal-to-noise, a combined-average line profile from about 1000 absorption lines in the spectrum. The LSD profile obtained in this way shows two distinct absorption profiles corresponding to the components of the binary, allowing us to measure the individual stellar radial velocities.

We determined an initial orbit solution to these radial velocity measurements using the BINARY code kindly provided by Gudehus, with the orbital period held fixed at the value determined from the photometric lightcurve (Sect. 2.1.2). To minimize the influence of epochs in which the cross-correlation functions show highly blended peaks, we excluded two epochs that occur within 0.05 phase of primary or secondary eclipse. The orbit solution was then iterated, and the internal error bars adjusted between iterations, until the final orbit solution yielded a reduced $\chi^{2}$ of unity. This orbit solution gives a best-fit eccentricity of $e=0.02 \pm 0.02$, i.e. formally consistent with a circular orbit. We then refined this solution by combining the radial velocity data with the observed lightcurve (see Sect. 3.3). This solution then yields: a binary mass ratio of $M_{1} / M_{2}=1.03 \pm 0.005$, velocity amplitudes of $K_{1}=92.4 \pm 0.33$ and $K_{2}=95.1 \pm 0.56$, and a system radial velocity of $\gamma=22.8 \pm 0.3 \mathrm{~km} \mathrm{~s}^{-1}$. The final radial velocity curve is shown in Fig. 4.

\subsection{Spectroscopic analysis}

In order to study each star individually, we numerically reconstructed the spectra of each of the two components with tomographic separation. This technique inverts the relation that the observed spectrum is a linear superposition of the spectra of the individual stars, where the only scaling factor is the luminosity ratio of the two components, which depends on their temperatures and radii. Provided that spectra observed at a range of phases are available, tomographic separation can efficiently reconstruct the individual stellar spectra, and is now a standard technique for the analysis of double-line spectrosocopic binaries (see, for example, Bagnuolo \& Gies 1991). We applied this technique to six spectra, selected on the basis of having the best signal-to-noise as well as covering a range of orbital phases. Here, we assumed a luminosity ratio of 2.0 for the two stars, which is consistent with the ratio of temperatures and radii obtained from lightcurve modelling (see Sect. 3.3).

From the individual component spectra we then determined the fundamental stellar parameters using the IDL-based spectroscopic analysis package SME (Valenti \& Piskunov 1996). SME combines radiative transfer calculations with multi-dimensional

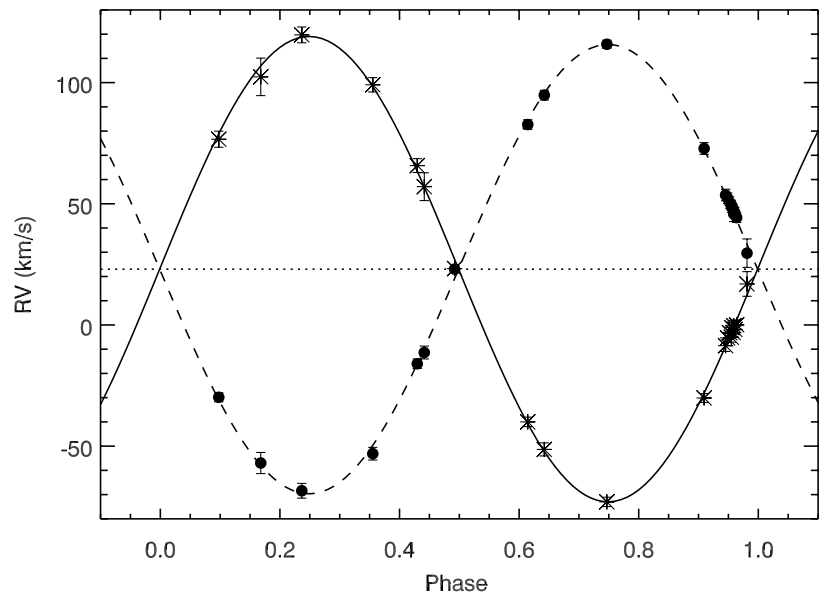

Fig. 4. Radial velocity measurements of the primary star (filled circles, dashed line) and secondary star (asteriks, solid line) of ASAS J052821+0338.5. A model radial velocity curve for both components is overplotted using the ephemeris derived in Sect. 2.1.2. A binary mass ratio, $M_{1} / M_{2}=1.03$, provides the best fit to the data.

least-squares minimization to determine which parameters (the effective temperature $T_{\text {eff }}$, the gravity $\log g$, the metallicity $[\mathrm{M} / \mathrm{H}]$, the projected rotational velocity $v \sin i$, the systemic radial velocity $v_{\text {rad }}$, the microturbulence $v_{\text {mic }}$ and the macroturbulence $v_{\text {mac }}$ ) best describe the observed stellar spectrum. The grid of model atmospheres used for radiative transfer are taken from Kurucz (1993), and atomic line data were obtained from the VALD database (Piskunov et al. 1995; Kupka et al. 1999). The atomic line data were checked and fine-tuned against a highquality spectrum of the Sun (Kurucz et al. 1984).

The two wavelength regions we considered in our spectrscopic analysis are 5880-5920 $\AA$ and 6000-6200 $\AA$. The first region contains the Na I D lines, sensitive to both $T_{\text {eff }}$ and $\log g$. The second regions contains a wealth of non-blended lines of different elements, providing constraints on $T_{\text {eff }}, \log g$ and $[\mathrm{M} / \mathrm{H}]$. We have no independent leverage on $v_{\text {mic }}$ and $v_{\text {mac }}$, and therefore assumed $v_{\text {mic }}=1.6 \mathrm{~km} \mathrm{~s}^{-1}$ (the average value for K-type PMS stars found by Padgett 1996) and $v_{\mathrm{mac}}=3.5 \mathrm{~km} \mathrm{~s}^{-1}$ (for a $T \approx 5000 \mathrm{~K}$ star, see Gray 1992). From this spectroscopic analysis we find for the primary $T_{\text {eff }}=5103 \pm 100 \mathrm{~K}$, $\log g=4.0 \pm 0.1$ and $[\mathrm{M} / \mathrm{H}]=-0.2 \pm 0.2$, and for the secondary $T_{\text {eff }}=4705 \pm 100 \mathrm{~K}, \log g=3.9 \pm 0.1$ and $[\mathrm{M} / \mathrm{H}]=-0.1 \pm 0.2$. These parameters correspond to spectral types of K1 for the primary and K3 for the secondary (Cohen \& Kuhi 1979).

The projected rotational velocities of the two components were determined by analysing the LSD line profiles (see Sect. 3.1) of each spectrum. During this process, we took into account line broadening from other sources, such as macroturbulence, smearing due to the orbital motion of the binary during the exposure and the instrumental profile. We find an average $v \sin i$ of $24.5 \pm 0.8 \mathrm{~km} \mathrm{~s}^{-1}$ and $24.5 \pm 0.7 \mathrm{~km} \mathrm{~s}^{-1}$ for, respectively, the primary and secondary. These values suggest the stars rotate synchronously (see Sect. 4).

In addition to the fundamental stellar parameters, we determined the abundance of lithium from the prominent resonance line at $6708 \AA\left(E W_{\text {prim }} \approx 307 \mathrm{~m} \AA, E W_{\text {sec }} \approx 394 \mathrm{~m} \AA\right)$. After applying corrections of -0.29 (primary) and -0.21 (secondary) for NLTE effects (Carlsson et al. 1994), we obtained $\log n(\mathrm{Li}) \equiv(\mathrm{Li} / \mathrm{H})+12=3.10 \pm 0.2$ for the primary, and $\log n(\mathrm{Li})=3.35 \pm 0.2$ for the secondary. These values are 


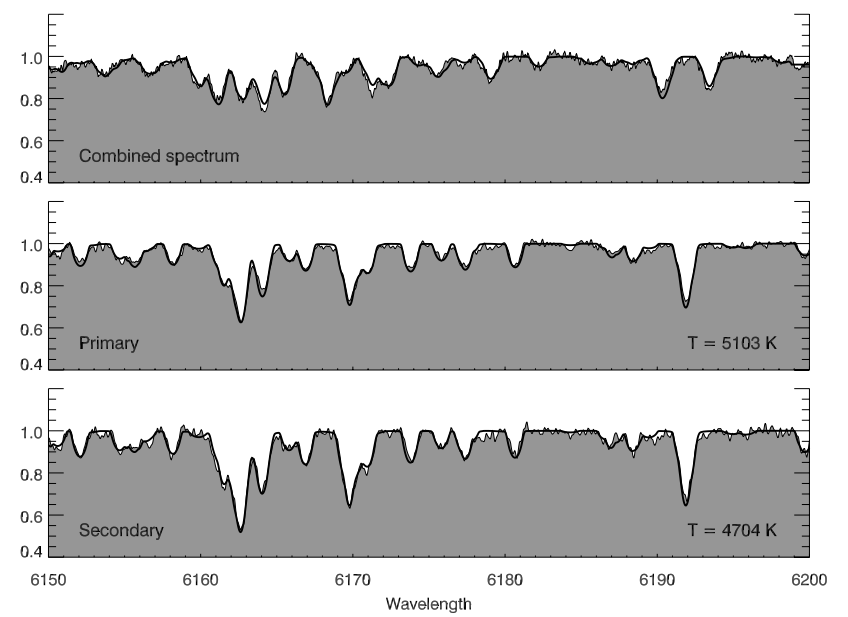

Fig. 5. The above panels show $50 \AA$ of the observed spectrum of ASAS J052821+0338.5 (upper panel), as well as the tomographically reconstructed spectra of the primary (middle panel) and the secondary component (lower panel), both in their respective stellar rest frames. The thick solid lines in each panel are synthetic spectra calculated with the stellar parameters from Table 1.

consistent with the commonly adopted value of 3.2-3.3 for the primordial lithium abundance.

Examples of tomographically separated spectra, as well as a comparison between the observed and synthetic spectra for each star are shown in Fig. 5. A full compilation of the stellar and atmospheric parameters is presented in Table 1 .

\subsection{Lightcurve modelling}

Together with determining the stellar and orbital parameters from spectroscopy we fit the $V$-band lightcurve and radial velocity data using the most recent version of the eclipsing binary lightcurve modeling algorithm of Wilson \& Devinney (1971, with updates), as implemented in the PHOEBE code of Prša \& Zwitter (2005). The code determines the surface gravity and effective temperature of each star based on the calculated gravitational potential, assuming full Roche geometry according to the formalism of Kopal (e.g. Wilson 1971), and the gravity brightening coefficients from Claret (2000). It includes theoretical Kurucz model atmospheres to determine intensities over the stellar disks. We adopted a linear limb darkening law using coefficients extrapolated down to $2700 \mathrm{~K}$ from Van Hamme (1993). We allowed the code to calculate reflection effects, adopting a bolometric albedo of 0.5 , typical for a fully convective stellar envelope. Since the out-of-eclipse variations in the observed lightcurve suggest the presence of starspots which are changing over time, we include in the modelling only $V$-band photometry obtained with the NMSU-1m and the JGT-1 $\mathrm{m}$ between January 9-February 10, 2007.

To begin, we first applied a traditional rectification procedure in which we fit the out-of-eclipse variations with a smooth polynomial function. The aim of this rectification procedure is to "remove" the spot signal from the lightcurve and to thereby allow the lightcurve model to achieve goodness of fit without introducing additional spot-fitting parameters that are poorly constrained at present.

Next, in order to maintain control of the lightcurve solution and its many free parameters, we performed this fitting in stages. In the initial stage we held the orbital parameters fixed at the values determined from the double-lined spectroscopic orbit
Table 1. Properties of ASAS J052821+0338.5. For each component we list the atmospheric parameters: effective temperature $\left(T_{\text {eff }}\right)$, surface gravity $(\log g)$, rotational velocity $(v \sin i)$, metallicity $([\mathrm{M} / \mathrm{H}])$ and lithium abundance $(\log n(\mathrm{Li}))$. We also present the system's ephemeris: the ephemeris reference epoch $\left(T_{0}\right)$ period $(P)$, eccentricity $(e)$, systemic radial velocity $(\gamma)$, inclination $(i)$ and projected semi-major axis $(a \sin i)$. Since the system's eccentricity is indistinguishable from truly circular, we choose $e=0$ and list no periastron angle or time for periastron passage. Finally, we list the parameters of our solutions from lightcurve modelling with and without spots: the velocity amplitude $(K)$, stellar mass $(M)$, radius $(R)$, luminosity $(L)$ and effective temperature $\left(T_{\text {eff }}\right)$.

\begin{tabular}{|c|c|c|c|}
\hline Parameter & Primary & Secondary & Unit \\
\hline \multicolumn{4}{|c|}{ Stellar parameters from spectral analysis } \\
\hline$T_{\text {eff }}$ & $5103 \pm 100$ & $4705 \pm 100$ & K \\
\hline $\log g$ & $4.0 \pm 0.1$ & $3.9 \pm 0.1$ & \multirow{4}{*}{$\mathrm{km} \mathrm{s}^{-1}$} \\
\hline$v \sin i$ & $24.5 \pm 0.8$ & $24.5 \pm 0.7$ & \\
\hline$[\mathrm{M} / \mathrm{H}]$ & $-0.2 \pm 0.2$ & $-0.1 \pm 0.2$ & \\
\hline $\log n(\mathrm{Li})$ & $3.10 \pm 0.2$ & $3.35 \pm 0.2$ & \\
\hline \multicolumn{4}{|c|}{ Parameters from rectified lightcurve modelling } \\
\hline$T_{0}$ & \multicolumn{2}{|c|}{ HJD $2454110.8500 \pm 0.005$} & \\
\hline$P$ & \multirow{2}{*}{\multicolumn{2}{|c|}{$\begin{array}{c}3.8729 \pm 0.0002 \\
0\end{array}$}} & $\mathrm{~d}$ \\
\hline$e$ & & & \\
\hline$\gamma$ & \multicolumn{2}{|c|}{$22.8 \pm 0.3$} & $\mathrm{~km} \mathrm{~s}^{-1}$ \\
\hline$i$ & \multicolumn{2}{|c|}{$83.7 \pm 1.0$} & $\operatorname{deg}$ \\
\hline$a \sin i$ & \multicolumn{2}{|c|}{$0.0668 \pm 0.0002$} & $\mathrm{AU}$ \\
\hline$K$ & $92.41 \pm 0.33$ & $95.08 \pm 0.56$ & $\mathrm{~km} \mathrm{~s}^{-1}$ \\
\hline$M$ & $1.375 \pm 0.011$ & $1.329 \pm 0.008$ & $M_{\odot}$ \\
\hline$R$ & $1.83 \pm 0.01$ & $1.73 \pm 0.01$ & $R_{\odot}$ \\
\hline$L$ & $2.05 \pm 0.16$ & $1.38 \pm 0.11$ & $L_{\odot}$ \\
\hline$T_{\text {eff }}$ & $5103^{*}$ & $4751 \pm 26$ & K \\
\hline $\log g$ & $4.05 \pm 0.01$ & $4.08 \pm 0.01$ & \\
\hline \multicolumn{4}{|c|}{ Parameters from EB modelling with spots } \\
\hline$M$ & $1.387 \pm 0.017$ & $1.331 \pm 0.011$ & $M_{\odot}$ \\
\hline$R$ & $1.84 \pm 0.01$ & $1.78 \pm 0.01$ & $R_{\odot}$ \\
\hline$L$ & $2.06 \pm 0.16$ & $1.28 \pm 0.10$ & $L_{\odot}$ \\
\hline$T_{\text {eff }}$ & $5103^{*}$ & $4599 \pm 23$ & $\mathrm{~K}$ \\
\hline $\log g$ & $4.05 \pm 0.02$ & $4.06 \pm 0.01$ & \\
\hline
\end{tabular}

* Assumed parameter for EB modelling.

solution (see Sect. 3.1), and fixed the effective temperature of the primary component to the value determined from our spectral analysis (5103 K; see Sect. 3.2). This allowed us to obtain initial estimates of the component temperatures and radii, and the system inclination.

With initial values for the temperatures, radii, and inclination so determined, we then iteratively improved the solution by first allowing the eccentricity and argument of periastron to be fit, and then performing a final fit in which all of the orbital and component parameters of the system were fit freely (except for the ephemeris, see Sect. 2.1.2). The eccentricity we recovered for the system, $e=0.007 \pm 0.006$, is indistinguishable from a circular orbit, and we could therefore not constrain the periastron angle. Also, if we assume a truely circular orbit $(e=0)$ we find no measurable change in any of the orbital parameters (within the limits of the error bars). We have therefore chosen to list this system as circular, and report no periastron angle or time for periastron passage. The final parameters that result from this simultaneous fit to the radial velocities and rectified lightcurve are presented in Table 1 .

To explore the influence of, and origin of the out-ofeclipse variability, and to better characterize systematic uncertainties in the stellar parameters arising from this variability, we have also modelled the un-rectified lightcurve by introducing 
multiple spots into the fitting analysis. The number of spots, their placement on the stellar surfaces, their temperatures and sizes, were initially determined manually in order to approximate the strong out-of-eclipse variability, then the code was permitted to adjust the spot parameters along with all of the other system and component parameters. We wish to emphasize that the adopted spot solution is neither definitive nor unique. Degenerate starspot configurations are consistent with the observed out-of-eclipse photometry, and it is not possible to break the degeneracy with the current single-band lightcurve. Therefore we present only one possible starspot configuration which well mimics the observed out-of-eclipse variations. Despite the degeneracy of the starspot solutions, the lightcurve cannot be adequately fit without the presence of a large $\left(\sim 25^{\circ}\right.$ radius $)$ cool spot on the back of the primary star. We also include a small hot spot near the back of the secondary star (i.e. facing away from the primary) in order to reproduce the small "bump" just prior to the primary eclipse. In Table 1 we summarize the stellar parameters resulting from simultaneously fitting the radial velocity and un-rectified lightcurve data, for ease of comparison with the parameters resulting from fitting the rectified lightcurve. The different treatment of modelling the distribution of starspots did no result in any change in the orbital parameters, and these are therefore not repeated in Table 1.

The stellar parameters resulting from the spot modelling procedure are overall in good agreement with those resulting from the rectification procedure. The formal errors on the stellar parameters derived from the above analysis are given in Table 1, but these do not include systematic errors inherent to the modelling procedures. Still, by comparing the results from the two methods (spotted vs. rectified) we find that, despite the obviously strong effect of spots on the lightcurve, a detailed treatment of the spots is evidently not critical for most of the stellar parameters of interest. The notable exceptions are the temperature and radius of the secondary. These parameters depend on the eclipse depths, which are affected by the manner in which the out-ofeclipse variations are modelled.

The temperature of the secondary is determined relative to the temperature of the primary (which we hold fixed at $5103 \mathrm{~K}$ ) by virtue of the temperature ratio, which is principally determined from the ratio of eclipse depths. Between the two methods of analysis, we find a difference of $\sim 150 \mathrm{~K}$ in the inferred temperature of the secondary, a difference that is significantly larger than the formal uncertainty of $\sim 25 \mathrm{~K}$ in the lightcurve model (Table 1). The temperature resulting from the rectified analysis is more consistent with the value from spectroscopic analysis of the tomographically separated spectra (Sect. 3.2), thus in what follows we adopt this solution as the preferred one. This lightcurve model is overplotted on the phase-folded data in Fig. 6.

The radii obtained from the two light-curve solutions also show larger differences than expected from the formal errors. Even so, the sum of the radii is consistent in both solutions to within $\sim 2 \%$. Due to the circular orbit, the primary and secondary eclipses are of equal durations and thus it is not possible to determine the individual radii independently from a single-band light curve. While the sum of the radii is very well constrained by the total duration of the two eclipses, almost any combination of component radii that satisfy the sum will yield a good fit to the light curve. Of course, the different component radii will in general result in very different flux ratios (since the temperature ratio is well constrained). Thus, we caution that additional observational constraints (e.g. flux ratios at multiple wavelengths) will be needed to firmly establish the individual radii accurately. The spectroscopically derived temperatures cannot by themselves constrain these flux ratios, since tomographic separation requires us to assume a luminosity ratio (see Sect. 3.2). We have therefore iteratively worked towards a solution for the temperatures and radii that is consistent with both the spectroscopic and the photometric models.

\subsection{Distance, physical location and kinematics}

We estimated the distance to ASAS J052821+0338.5 by comparing distance moduli from the available broad-band magnitudes. For this analysis, we have assumed relatively large uncertainties on the observed magnitudes $\left(0.15^{\mathrm{m}}\right)$, because we have no possibility of knowing whether these are truly out-of-eclipse values. Using the components' surface temperatures of the preferred solution from lightcurve modelling (5103 K and 4751 K) and the standard color indices tabulated by Kenyon \& Hartmann (1995), we find that the distance moduli of the available colors converge on a distance of $280 \pm 30 \mathrm{pc}$ and an extinction of $A_{V}=0.6 \pm 0.3^{\mathrm{m}}$. The distance is mostly constrained by the (almost) extinction-insensitive $J, H$ and $K$ magnitudes, while the extinction depends mostly on the $B$ and $V$ magnitudes. The quoted error margins are dominated by the uncertainties of the observed magnitudes.

In addition to the above, we compared the observed broadband magnitudes of ASAS J052821+0338.5 with model stellar atmosphere spectra from Castelli \& Kurucz (2003), using the SED fitting routines described in Robitaille et al. (2007; modified to incorporate stellar atmosphere models). Assuming a temperature of $5000 \mathrm{~K}$, which is the luminosity-weighted average temperature of the binary, we find the broad-band magnitudes to be compatible with an extinction of $A_{V} \approx 0.65^{\mathrm{m}}$. We also measured the equivalent width of the narrow interstellar absorption components in the Na I D1 line. We find two absorption components with a combined $E W$ of about $200 \mathrm{~m} \AA$, which translates to $A_{V} \approx 0.25 \pm 0.1^{\mathrm{m}}$ (Munari \& Zwitter 1997). The interstellar Na I D absorption is partly filled-in by telluric emission, and therefore this value should be considered a lower limit to $A_{V}$. We notice that the strongest absorption component (with an $E W$ of $\sim 150 \mathrm{~m} \AA$ ) has a central velocity equal to the systemic velocity of the system, suggesting that a large part of the extinction originates from regions close to the binary.

Following the definitions of Warren \& Hesser (1977), ASAS J052821+0338.5 is located in the direction of the widely spread Orion OB1a subassociation. The system is about 3-4 degrees away from the much more compact Orion OB1b subassociation and about 2 degrees from the 25 Ori subassociation, a compact and kinematically distinct subgroup of Orion OB1a recently identified by Briceño et al. (2007). Orion OB1a is located at a distance of $330 \pm 15 \mathrm{pc}$, while Orion OB1b is further away, at a distance $440 \pm 20$ pc (Brown et al. 1994; de Zeeuw et al. 1999; Hernández et al. 2005). There is little difference in the kinematic distribution of Orion OB1a and Orion OB1b. The average radial velocities of Orion OB1a and Orion OB1b, $23.8 \pm 0.7 \mathrm{~km} \mathrm{~s}^{-1}$ and $23.1 \pm 1.4 \mathrm{~km} \mathrm{~s}^{-1}$ respectively (Morrell \& Levato 1991), and also their tangential velocity distributions are similar $\left(\mu_{\alpha}=0.44 \pm 0.25 \mathrm{mas} / \mathrm{yr}, \mu_{\delta}=-0.65 \pm 0.25 \mathrm{mas} / \mathrm{yr}\right.$, Brown et al. 1998; de Zeeuw et al. 1999). Orion OB1a is commonly assumed to be $11.4 \pm 1.9$ Myr old, significantly older than the 1.7 \pm 1.1 Myr old Orion OB1b (Brown et al. 1994).

The systemic radial velocity we determined for ASAS J052821+0338.5 is $22.8 \pm 0.3 \mathrm{~km} \mathrm{~s}^{-1}$, and the tangential velocity from the NOMAD catalog is $\mu_{\alpha}=-1.6 \pm 2.8 \mathrm{mas} / \mathrm{yr}$, and $\mu_{\delta}=-4.0 \pm 4.0 \mathrm{mas} / \mathrm{yr}$. These kinematics combined 


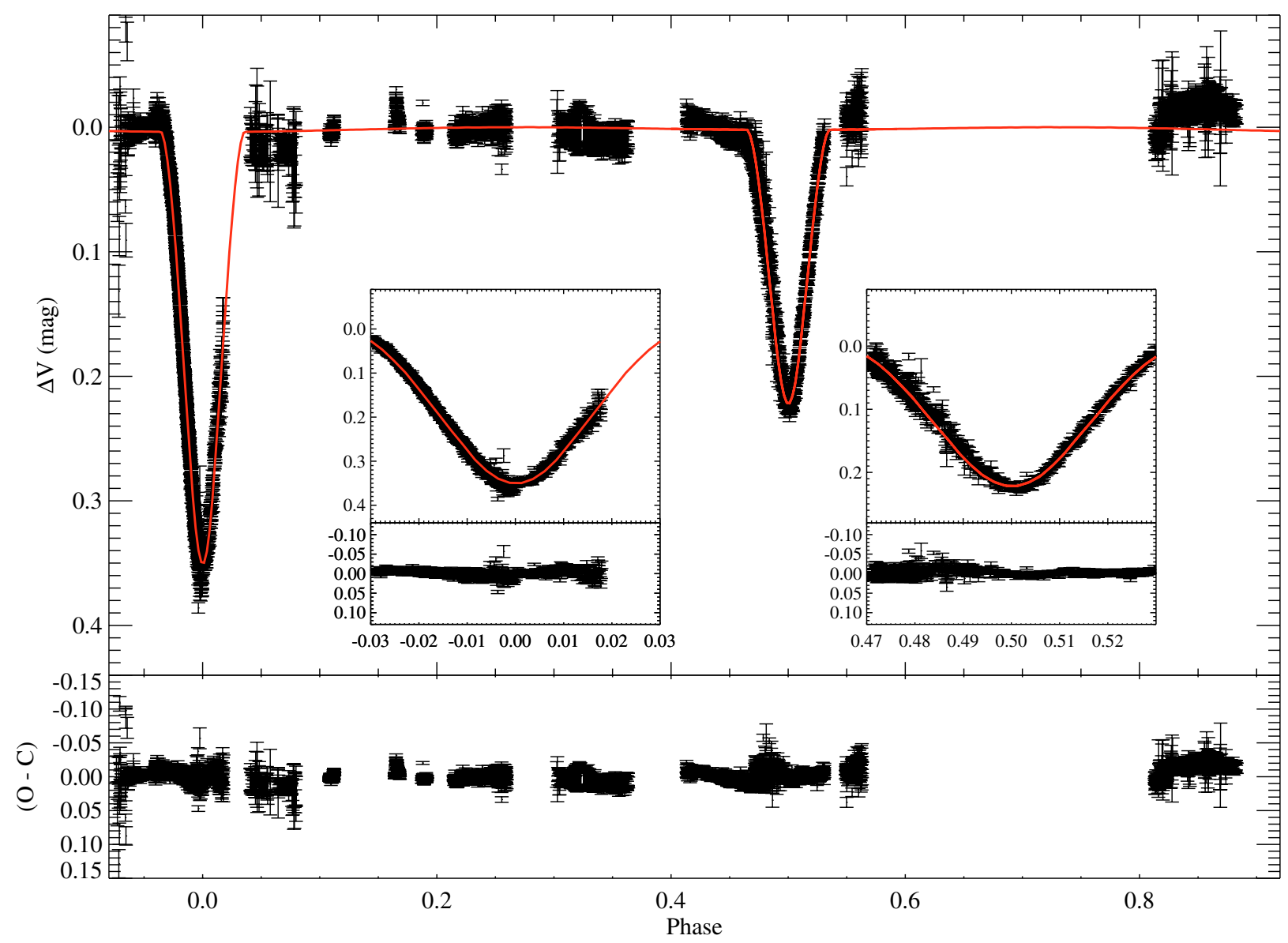

Fig. 6. The top panel shows the phase-folded and rectified differential $V$-band lightcurve obtained with the NMSU-1 m and JGT-1 m telescopes. The lightcurve has a precision of $0.005-0.01^{\mathrm{m}}$ and a sampling of $<1 \mathrm{~min}$. The data are phase-folded with a period, $P=3.8729$ days, and epoch, $T_{0}=$ HJD 2454110.8500 , as well as rectified by fitting a smooth polynomial to the out-of-eclipse variations. The smooth grey curve shows the calculated binary lightcurve that has the best agreement with the data. The residuals of this curve with the data are shown in the bottom panel.

with the estimated distance and physical location suggest that ASAS J052821+0338.5 is most probably a member of the $\sim 11$ Myr old Orion OB1a subassociation.

\subsection{Evolutionary status}

Several characteristics of the two binary components, including their large radii, their cool temperatures, and their primordial lithium abundances, as well as the proximity to the Orion OB1a subassociation strongly indicate that ASAS J052821+0338.5 is a young, pre-main sequence eclipsing binary.

In Fig. 7, the structural properties of the binary are shown in relation to other PMS and main-sequence eclipsing binaries, as well as theoretical stellar evolution models from Baraffe et al. (1998). Both components have larger radii than mainsequence objects of the same mass, as expected for stars that are still evolving onto the main sequence. However, their large observed radii do not alone imply a pre-main-sequence evolutionary state. A typical, post-main-sequence, $1.4 M_{\odot}$ star with solar metallicity reaches a radius similar to that of the primary component of ASAS J052821+0338.5 at an age of $\sim 2$ Gyr. Yet, at about $6000 \mathrm{~K}$, such a star is considerably hotter than either component of ASAS J052821+0338.5. A $1.4 M_{\odot}$ postmain-sequence star will cool to a temperature comparable with ASAS J052821+0338.5 ( 5000 K) as it evolves through the red giant phase, but this necessarily coincides with a further increase in radius to a value much larger than what is observed in the binary. Thus, the cool temperatures of the binary components combined with their $\sim 1.4 M_{\odot}$ masses and $<2 R_{\odot}$ radii suggest ASAS J052821+0338.5 is a pre-main sequence object.

This is exemplified in the modified Hertzprung-Russel (HR) diagram shown in Fig. 8. In this figure, stellar radius is a proxy for luminosity, as it is a direct measurement from the eclipsing binary analysis, and it relates to luminosity through the temperature. Evolutionary tracks and isochrones for the Baraffe et al. (1998) models are shown in addition to the position of ASAS J052821+0338.5 and the other known PMS eclipsing binaries. We interpolated the evolutionary tracks at the masses of the primary (black solid line) and secondary (black dashed line) components. ASAS J052821+0338.5 is clearly positioned in the PMS phase of evolution in this parameter space when compared to empirical data and to this set of models.

A further indication of the PMS evolutionary state of ASAS J052821+0338.5 is the detection of primordial lithium in both components. The rate of lithium depletion in late-type stars depends on the depth of the convection zone (and thus, on temperature), and the primary is therefore expected to have the slowest lithium depletion rate. The primary, with an effective temperature of $5100 \mathrm{~K}$, will deplete lithium well below the primordial level after about 150-200 Myr (Sestito \& Randich 2005). In conclusion, only very young stars can have bloated radii, cool temperatures, and primordial lithium.

Finally, although determining an absolute age of ASAS J052821+0338.5 would involve a comparison with 


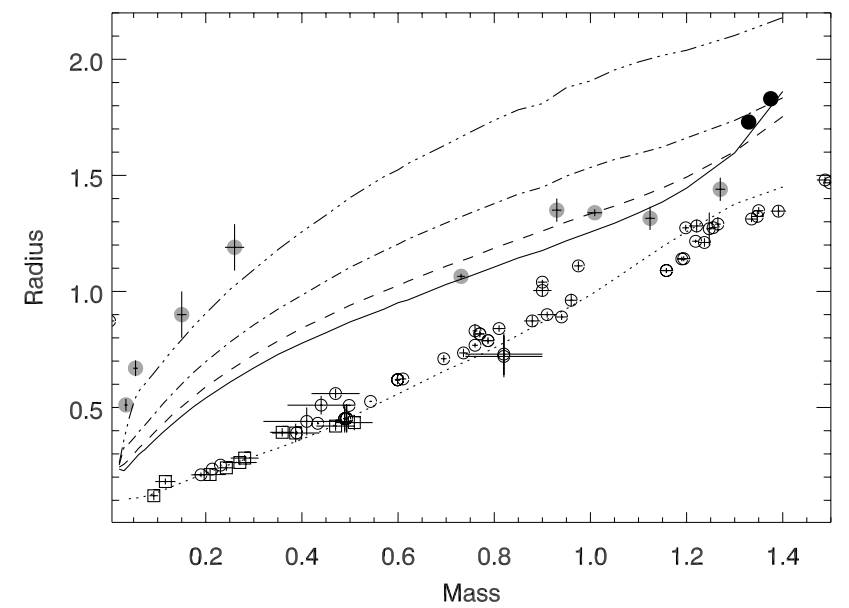

Fig. 7. Mass versus radius for known main sequence (open circles) and pre-main sequence (grey solid circles) eclipsing binaries. The components of ASAS J052821+0338.5 are shown as black solid circles. Isochrones from Baraffe et al. (1998) with ages of $3 \mathrm{Myr}, 6 \mathrm{Myr}, 10 \mathrm{Myr}$, $13 \mathrm{Myr}$, and $300 \mathrm{Myr}$ are overplotted.

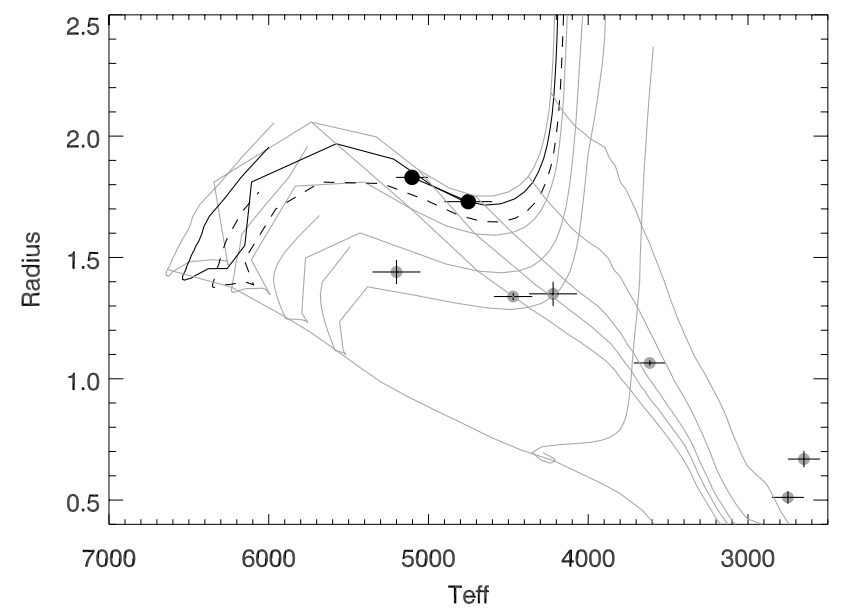

Fig. 8. The modified Hertzprung-Russel diagram showing radius versus effective temperature for the components of ASAS J052821+0338.5 (black solid circles) and other PMS EBs in Orion (grey solid circles). Evolutionary tracks $\left(1.4,1.3,1.2,1.1,0.7 M_{\odot}\right)$ and isochrones $(3,6$, 10, 13, 16, $300 \mathrm{Myr}$ ) from Baraffe et al. 1998 are overplotted as grey lines. The black solid line shows the evolutionary track interpolated at the mass of the primary star $\left(1.375 M_{\odot}\right)$. The black dashed line shows the evolutionary track interpolated at the mass of the secondary star $\left(1.329 M_{\odot}\right)$.

largely uncalibrated stellar evolutionary models, we do note that the fundamental parameters of ASAS J052821+0338.5 are consistent with the 10-13 Myr solar-metallicity isochrones of Baraffe et al. (1998). This is in agreement with the age of the Orion OB1a subassociation (11.4 \pm 1.9 Myr, see Sect. 3.4).

\section{Discussion}

The photometric and spectroscopic observations we obtained of ASAS J052821+0338.5 identify this system as a new pre-mainsequence eclipsing binary with a period of 3.87 days. The masses and radii of the components place the system in an as yet unsampled region of the mass-radius and HR diagrams, and thereby provide a new pair of reference points for PMS stellar evolutionary models. Both components of the system are more massive than all other members of known PMS eclipsing systems, except for TY CrA B, the $1.64 M_{\odot}$ and $\sim 3$ Myr old companion to a main-sequence B-type primary (Casey et al. 1998).

The physical location and kinematic properties of ASAS J052821+0338.5 suggest this system to be a member of the $\sim 11.4 \pm 1.9$ Myr Orion OB1a subassociation. This age is also compatible with the position of the two components in the mass-radius diagram shown in Fig. 7, where both stars fall close to the $13 \mathrm{Myr}$ isochrone of the Baraffe et al. (1998) evolutionary models. At this age, the two $1.35 M_{\odot}$ stars have just left the fully-convective Hayashi stage. The temperature of the two components will then increase at almost constant luminosity, first slightly growing in radius before their final contraction towards the main sequence, settling at a final temperature of $\sim 6250 \mathrm{~K}$ (for a more detailed description of pre-main-sequence evolution, see for example Palla \& Stahler 1993). We also find a that the locations of the stars in the HR diagram agree quite well with the predicted 13 Myr evolutionary tracks of Baraffe et al. (1998, see Fig. 8). Although there appear to be small differences between the observed radii and the predicted values, this does not indicate any inconsistency since the only well-constrained parameter is the sum of the radii (see Sect. 3.3), and full consistency of both stars with the predicted tracks requires only small antagonal adjustements (of about 3\%) to the stellar radii. Therefore, a critical next step to further improve these parameters will be a multi-band lightcurve analysis with a more detailed spot treatment.

Most of the known PMS eclipsing binaries are located in Orion. ASAS J052821+0338.5 and RXJ 0529.4+0041A are thought to be located in the Orion OB1a subassociation (Covino et al. 2000, 2004). V1174 Ori is considered to be a member of Orion OB1c (Stassun et al. 2004) and 2MASS J0535218420130546085, JW 380 and Par 1802 are found in the Orion Nebula Cluster (Stassun et al. 2006; Irwin et al. 2007b; Cargile et al. 2007). These OB subassociations in Orion form an evolutionary sequence, with ages of $\sim 1-12 \mathrm{Myr}$. This age range covers a key period in star formation when stars experience rapid evolution in both radius and temperature. The components of the PMS eclipsing binaries in Orion have masses between 0.03 and $1.35 M_{\odot}$ and have well-determined radii and temperatures. As such they form an easily accessible and homogenous sample of late-type PMS stars that can be used to perform relative calibrations of PMS stellar evolutionary models.

As described in Sect. 2.1.1, we detected considerable $\left(\sim 0.15^{\mathrm{m}}\right)$ out-of-eclipse variations, occuring around the same orbital phase throughout our four-month campaign of photometric observations, not unlike the "photometric wave" that is characteristic of the chromospherically active RS CVn binaries (see the review by Rodonó 1992). Like in an RS CVn binary, the components of ASAS J052821+0338.5 have relatively short rotational periods and extensive convective envelopes, which help to drive a stellar dynamo and enhance magnetic activity. The photometric variations may then be explained by a large cool spot (or region of spots) located on the back of the primary, rotating in and out of view in phase with the binary period. However, this configuration requires the primary to rotate synchronously with the binary orbit. According to the theory of Zahn \& Bouchet (1989), tidal breaking during the Hayashi phase, when stars are fully convective, is a very efficient mechanism for inducing orbital synchronization and circularization in short-period latetype binaries. Once the convective envelope starts decreasing, tidal breaking loses its efficiency, and further stellar contraction may lead to (temporary) departures from synchronous rotation. ASAS J052821+0338.5 seems to follow this pattern. The orbit is almost perfectly circularized, and the observed rotational 
velocities of the primary and secondary are $24.5 \pm 0.8 \mathrm{~km} \mathrm{~s}^{-1}$ and $24.5 \pm 0.7 \mathrm{~km} \mathrm{~s}^{-1}$, respectively. Since the expected synchronous rotational velocities are $24.0 \mathrm{~km} \mathrm{~s}^{-1}$ and $23.4 \mathrm{~km} \mathrm{~s}^{-1}$, the primary is most likely in synchronous rotation with the binary orbit, while the smaller secondary may rotate slightly supersynchronously.

While an extended cool spot on the back of the primary can fully explain the observed out-of-eclipse photometric variations, we did not detect any signatures of a cool spot in our current high-resolution spectra, such as changes in the observed line-ratios or the appearance of molecular features. However, the brightness contrast between the spotted and the unspotted areas is large, and since the signal-to-noise of our spectra is moderate it is unclear whether such an effect would be detectable. An alternative explanation to the out-of-eclipse variability might be periodic eclipses of the system by a warped circumbinary disk, or by circumstellar material in co-rotation with the binary. Such concentrations of circumstellar matter localized around the stable co-rotating Lagrangian points (L2 and L3) have been detected in the close PMS binary V4046 Sgr (Stempels \& Gahm 2004). We are currently pursuing multi-band photometry of the system in order to determine the nature of the out-of-eclipse variations, as well as to further improve the orbital and stellar parameters of ASAS J052821+0338.5, in particular the temperatures and radii of the components.

Acknowledgements. We thank Jonathan Irwin for obtaining photometry with the Cambridge $0.5 \mathrm{~m}$ 3-Mirror Telescope. HCS acknowledges support from the Swedish Research Council. KGS gratefully acknowledges support from National Science Foundation Career grant AST-0349075. We would like to thank an anomymous referee for the comments and questions that helped to improve this paper. The Nordic Optical Telescope is operated on the island of La Palma jointly by Denmark, Finland, Iceland, Norway, and Sweden, in the Spanish Observatorio del Roque de los Muchachos of the Instituto de Astrofisica de Canarias.

\section{References}

Andersen, J. 1991, A\&ARv, 3, 91

Bagnuolo, W. G., Jr., \& Gies, D. R. 1991, ApJ, 376, 266

Baraffe, I., Chabrier, G., Allard, F., \& Hauschildt, P. H. 1998, A\&A, 337, 403

Briceño, C., Hartmann, L., Hernández, J., et al. 2007, ApJ, 661, 1119

Brown, A. G. A., de Geus, E. J., \& de Zeeuw, P. T. 1994, A\&A, 289, 101

Brown, A. G. A., Walter, F. M., \& Blaauw, A. 1998 (contribution to the unpublished proceedings of the workshop The Orion Complex Revisited) [arXiv: astro-ph/9802054]

Cargile, P., Stassun, K. G., \& Mathieu, R. 2007, ApJ, in press

Carlsson, M., Rutten, R. J., Bruls, J. H. M. J., \& Shchukina, N. G. 1994, A\&A, 288,860
Casey, B. W., Mathieu, R. D., Vaz, L. P. R., Andersen, J., \& Suntzeff, N. B. 1998, AJ, 115,1617

Castelli, F., \& Kurucz, R. L. 2003, in Modelling Stellar Atmospheres, ed. N. Piskunov, W. W. Weiss, \& D. F. Gray, Proc. IAU Symp., 210, A20

Claret, A. 2000, A\&A, 363, 1081

Cohen, M., \& Kuhi, L. V. 1979, ApJS, 41, 743

Covino, E., Catalano, S., Frasca, A., et al. 2000, A\&A, 361, L49

Covino, E., Frasca, A., Alcalá, J. M., Paladino, R., \& Sterzik, M. F. 2004, A\&A, 427, 637

de Zeeuw, P. T., Hoogerwerf, R., de Bruijne, J. H. J., Brown, A. G. A., \& Blaauw, A. 1999, AJ, 117, 354

Donati, J.-F., Semel, M., Carter, B. D., Rees, D. E., \& Collier Cameron, A. 1997, MNRAS, 291, 658

Gray, D. F. 1992, The Observation and Analysis of Stellar Photospheres (Cambridge University Press), 2nd ed.

Hernández, J., Calvet, N., Hartmann, L., et al. 2005, AJ, 129, 856

Irwin, M., \& Lewis, J. 2001, NewAR, 45, 105

Irwin, J., Irwin, M., Aigrain, S., et al. 2007, MNRAS, 375, 1449

Irwin, J., et al. 2007, MNRAS, in press [arXiv:0706.2325]

Kenyon, S. J., \& Hartmann, L. 1995, ApJS, 101, 117

Kupka, F., Piskunov, N., Ryabchikova, T. A., Stempels, H. C., \& Weiss, W. W. 1999, A\&AS, 138, 119

Kurucz, R. 1993, ATLAS9 Stellar Atmosphere Programs and $2 \mathrm{~km} \mathrm{~s}^{-1}$ grid, Kurucz CD-ROM No. 13 (Cambridge, Mass.: Smithsonian Astrophysical Observatory)

Kurucz, R. L., Furenlid, I., Brault, J., \& Testerman, L. 1984, NSO Atlas, Sunspot (New Mexico: National Solar Observatory)

Morrell, N., \& Levato, H. 1991, ApJS, 75, 965

Munari, U., \& Zwitter, T. 1997, A\&A, 318, 269

Padgett, D. L. 1996, ApJ, 471, 847

Palla, F., \& Stahler, S. W. 1993, ApJ, 418, 414

Piskunov, N. E., Kupka, F., Ryabchikova, T. A., Weiss, W. W., \& Jeffery, C. S. 1995, A\&AS, 112, 525

Pojmanski, G. 2002, Acta Astron., 52, 397

Popper, D. M. 1980, ARA\&A, 18, 115

Popper, D. M. 1987, ApJ, 313, L81

Prša, A., \& Zwitter, T. 2005, ApJ, 628, 426

Robitaille, T. P., Whitney, B. A., Indebetouw, R., \& Wood, K. 2007, ApJS, 169, 328

Rodonò, M. 1992, in Evolutionary Processes in Interacting Binary Stars, ed. Y. Kondo, R. Sistero, \& R. S. Polidan, Proc. IAU Symp., 151, 71

Sestito, P., \& Randich, S. 2005, A\&A, 442, 615

Stassun, K. G., Mathieu, R. D., \& Valenti, J. A. 2006, Nature, 440, 311

Stassun, K. G., Mathieu, R. D., Vaz, L. P. R., Stroud, N., \& Vrba, F. J. 2004, ApJS, 151, 357

Stassun, K. G., Mathieu, R. D., \& Valenti, J. A. 2007, ApJ, 664, 1154

Stempels, H. C., \& Gahm, G. F. 2004, A\&A, 421, 1159

Udry, S., Mayor, M., \& Queloz, D. 1999, IAU Colloq. 170: Precise Stellar Radial Velocities, ASP Conf. Ser., 185, 367

Valenti, J. A., \& Piskunov, N. 1996, A\&AS, 118, 595

van Hamme, W. 1993, AJ, 106, 2096

Warren, W. H., Jr., \& Hesser, J. E. 1977, ApJS, 34, 115

Wilson, R. E. 1979, ApJ, 234, 1054

Wilson, R. E., \& Devinney, E. J. 1971, ApJ, 166, 605

Zacharias, N., Monet, D. G., Levine, S. E., et al. 2004, BAAS, 36, 1418

Zahn, J.-P., \& Bouchet, L. 1989, A\&A, 223, 112 